OPEN ACCESS

Edited by:

Jorge Matias-Guiu,

Complutense University of Madrid,

Spain

Reviewed by:

Ulises Gomez-Pinedo,

Instituto de Investigación Sanitaria del

Hospital Clínico San Carlos, Spain

Matteo Gastaldi,

Neurological Institute Foundation

Casimiro Mondino (IRCCS), Italy

*Correspondence:

Gustavo C. Román

gcroman@houstonmethodist.org

${ }^{\dagger}$ These authors have contributed equally to this work

Specialty section: This article was submitted to Multiple Sclerosis and Neuroimmunology, a section of the journa

Frontiers in Immunology

Received: 15 January 2021 Accepted: 08 March 2021

Published: 26 April 2021

Citation:

Román GC, Gracia F, Torres A, Palacios A, Gracia K and Harris $D$

(2021) Acute Transverse Myelitis (ATM):Clinical Review of 43 Patients With COVID-19-Associated ATM and

3 Post-Vaccination ATM Serious Adverse Events With the ChAdOx1 $n$ CoV-19 Vaccine (AZD1222).

Front. Immunol. 12:653786. doi: 10.3389/fimmu.2021.653786

\section{Acute Transverse Myelitis (ATM): Clinical Review of 43 Patients With COVID-19-Associated ATM and 3 Post-Vaccination ATM Serious Adverse Events With the ChAdOx1 nCoV-19 Vaccine (AZD1222)}

\author{
Gustavo C. Román ${ }^{1,2,3 *+}$, Fernando Gracia ${ }^{4,5,6 t}$, Antonio Torres ${ }^{7}$, Alexis Palacios ${ }^{8}$, \\ Karla Gracia ${ }^{9}$ and Diógenes Harris ${ }^{10}$ \\ ${ }^{1}$ Department of Neurology, Neurological Institute, Houston Methodist Hospital, Houston, TX, United States, ${ }^{2}$ Weill Cornell \\ College of Medicine, Cornell University, New York, NY, United States, ${ }^{3}$ Department of Neurology, Texas A\&M University College \\ of Medicine, Bryan, TX, United States, ${ }^{4}$ Neurology Service, Hospital Paitilla, Panama City, Panama, ${ }^{5}$ Faculty of Health Sciences, \\ Interamerican University of Panama, Panama City, Panama, ${ }^{6}$ Neurology Service, Hospital Santo Tomás, Panama City, Panama, \\ 7 Infectious Disease Service, Hospital Santo Tomás, Panama City, Panama, ${ }^{8}$ Neuroradiology Service, Complejo Hospitalario \\ Metropolitano, CSS (Caja de Seguro Social), Panama City, Panama, 9 Interamerican University of Panama, Panama City, \\ Panama, ${ }^{10}$ Neurosurgery Service, Complejo Hospitalario Metropolitano, CSS, Panama City, Panama
}

Introduction: Although acute transverse myelitis (ATM) is a rare neurological condition (1.34-4.6 cases per million/year) COVID-19-associated ATM cases have occurred during the pandemic.

Case-finding methods: We report a patient from Panama with SARS-CoV-2 infection complicated by ATM and present a comprehensive clinical review of 43 patients with COVID-19-associated ATM from 21 countries published from March 2020 to January 2021. In addition, 3 cases of ATM were reported as serious adverse events during the clinical trials of the COVID-19 vaccine ChAdOx1 nCoV-19 (AZD1222).

Results: All patients had typical features of ATM with acute onset of paralysis, sensory level and sphincter deficits due to spinal cord lesions demonstrated by imaging. There were 23 males (53\%) and 20 females (47\%) ranging from ages 21- to 73-years-old (mean age, 49 years), with two peaks at 29 and 58 years, excluding 3 pediatric cases. The main clinical manifestations were quadriplegia (58\%) and paraplegia (42\%). MRI reports were available in 40 patients; localized ATM lesions affected $\leq 3$ cord segments (12 cases, $30 \%$ ) at cervical (5 cases) and thoracic cord levels (7 cases); 28 cases (70\%) had longitudinallyextensive ATM (LEATM) involving $\geq 4$ spinal cord segments (cervicothoracic in 18 cases and thoracolumbar-sacral in 10 patients). Acute disseminated encephalomyelitis (ADEM) occurred in 8 patients, mainly women (67\%) ranging from 27 - to 64 -years-old. Three ATM patients also had blindness from myeloneuritis optica (MNO) and two more also had acute motor axonal neuropathy (AMAN). 
Conclusions: We found ATM to be an unexpectedly frequent neurological complication of COVID-19. Most cases (68\%) had a latency of 10 days to 6 weeks that may indicate post-infectious neurological complications mediated by the host's response to the virus. In 32\% a brief latency (15 hours to 5 days) suggested a direct neurotropic effect of SARSCoV-2. The occurrence of 3 reported ATM adverse effects among 11,636 participants in the AZD1222 vaccine trials is extremely high considering a worldwide incidence of $0.5 /$ million COVID-19-associated ATM cases found in this report. The pathogenesis of ATM remains unknown, but it is conceivable that SARS-CoV-2 antigens -perhaps also present in the AZD1222 COVID-19 vaccine or its chimpanzee adenovirus adjuvant- may induce immune mechanisms leading to the myelitis.

Keywords: COVID-19, neurological complications, SARS-CoV-2 neurotropism, myelitis, transverse myelitis, COVID-19 ChAdOx1 nCoV-19 vaccine

\section{INTRODUCTION}

Neurological complications of coronavirus disease 2019 (COVID-19) are well recognized (1-3) and affect both the central nervous system (CNS) and the peripheral nervous system (PNS). Neurological injury results from the affinity of the COVID-19 etiological agent, the Severe Acute Respiratory Syndrome coronavirus 2 (SARS-CoV-2), for the angiotensin-converting enzyme 2 (ACE2) receptor present in neurons and glial cells endowing high neuroinvasive potential to SARS-CoV-2 compared to previous coronaviruses. The high frequency of anosmia during the acute infection probably reflects viral invasion of the olfactory bulbs. Cells with abundant ACE2 receptors are infected first by this coronavirus including nasal epithelium cells, ciliated bronchial epithelial cells and type II pneumocytes, explaining the severity of the pulmonary involvement. Also, the presence of ACE2 receptors for the viral S protein in endothelial cells correlates with the frequent vascular complications of COVID-19 resulting from endotheliitis and microvascular brain injury (4) that induces the host's immune response with cytokine storm, hyperinflammation, coagulopathy, thrombosis and embolism resulting in ischemic and hemorrhagic strokes and multisystemic complications affecting lungs, heart, kidneys and liver.

According to Borchers and Gershwin (5), ATM is a rare neurological condition in adults with an estimated incidence ranging between 1.34 and 4.6 cases per million annually with a mean age of 3540 years. We report a patient with SARS-CoV-2 infection in Panama who developed acute transverse myelitis (ATM) and we present the results of a comprehensive review of COVID-19-associated myelitis that yielded 42 additional cases reported in 21 countries worldwide (6-44) published from March 2020 to January 2021 during year 1 of the pandemic (Table 1 and Supplementary Table 1A). Furthermore, 3 ATM serious adverse events were reported with the ChAdOx1 $\mathrm{nCoV}-19$ (AZD1222) vaccine trials $(45,46)$.

\section{CASE DESCRIPTION}

A previously-healthy 72 -year-old man presented to the emergency department at a hospital in Panama City, Panama, complaining of sudden difficulty to urinate. The urologist diagnosed neurogenic bladder and placed a Foley catheter. Three days later the patient developed dysesthesias in arms and legs and weakness of all four limbs. Neurologic examination showed $3+/ 5$ strength in the upper extremities and $1+/ 5$ in the lower limbs with spastic paraplegia, generalized hyperreflexia, bilateral Babinski, and spontaneous pyramidal jerking of both legs; sensory examination disclosed decreased proprioception in the legs and a tactile sensory level below $\mathrm{Th}_{9}$. The patient was alert and oriented; higher cortical functions, cranial nerves and cerebellar examination were all intact.

He denied fever, headache, ageusia, anosmia, fatigue, diarrhea or upper respiratory symptoms during the past 3 weeks. Past medical history was negative except for hypertension controlled with enalapril. The SARS-CoV-2 RNA PCR nasal swab test was negative on 2 occasions. His wife was asymptomatic, but her nasal swab test was positive, and she had SARS-CoV-2 antibodies. The patient's serology demonstrated recent infection with SARS-CoV-2 IgG index $=3.53$ (normal <1.6) and IgM index $=5.1$ (normal $<0.6)$. Chest $\mathrm{X}$-rays showed mild cardiomegaly but no evidence of consolidation or pleural effusion. Chest computerized tomography (CT) scan was normal. Electrocardiogram showed mild left ventricular hypertrophy. The patient was afebrile and his general physical evaluation was normal. Respiratory rate 16 breaths per minute, oxygen saturation $98 \%$ on room air, blood pressure $130 / 80$ $\mathrm{mmHg}$, heart rate 78 beats per minute and temperature $36.8^{\circ} \mathrm{C}$.

Laboratory results showed normal white blood cell count $(8,100 / \mu \mathrm{L})$ with normal hemoglobin $(14.7 \mathrm{~g} / \mathrm{dl})$. Inflammatory markers showed elevated C-reactive protein at $1.7 \mathrm{mg} / \mathrm{dl}$ and high erythrocyte sedimentation rate at $51 \mathrm{~mm} / \mathrm{hr}$ (normal range $0-10 \mathrm{~mm} / \mathrm{hr}$ ). Coagulation profile was normal. Protein C, Protein S, Antithrombin III, and activated Protein C resistance were within normal limits. Hematology consult found no associated pathology. Hepatic and thyroid function tests were normal. VDRL and HIV tests were negative. Autoimmune immunological screening was negative for lupus anticoagulant, anti-nuclear antibodies (ANA), anti-neutrophil cytoplasmic antibodies, rheumatoid factor, anti-cardiolipin, and 
TABLE 1 | Summary of SARS-CoV-2-Associated Myelitis Published Cased from March-2020 until January-2021.

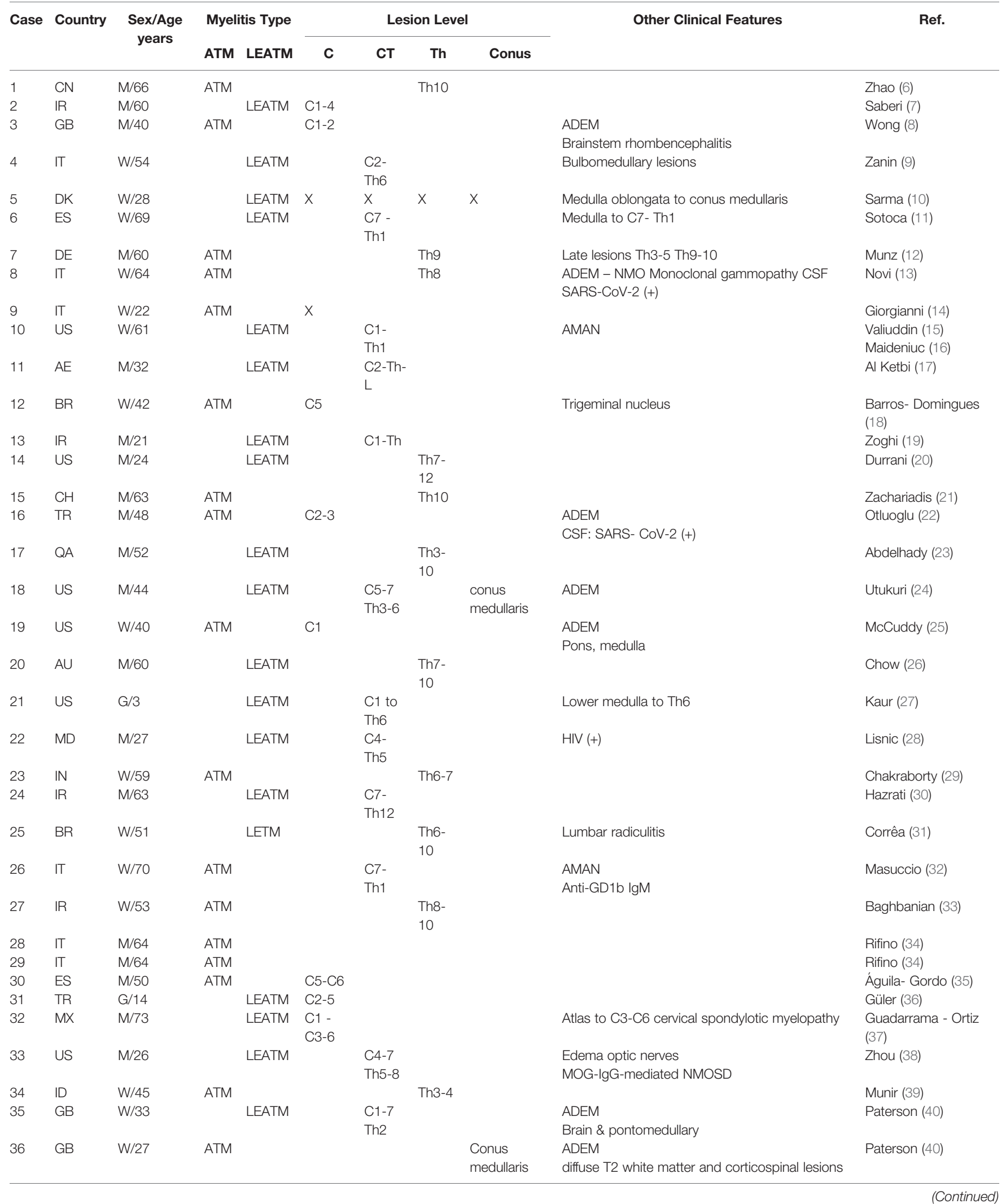


TABLE 1 | Continued

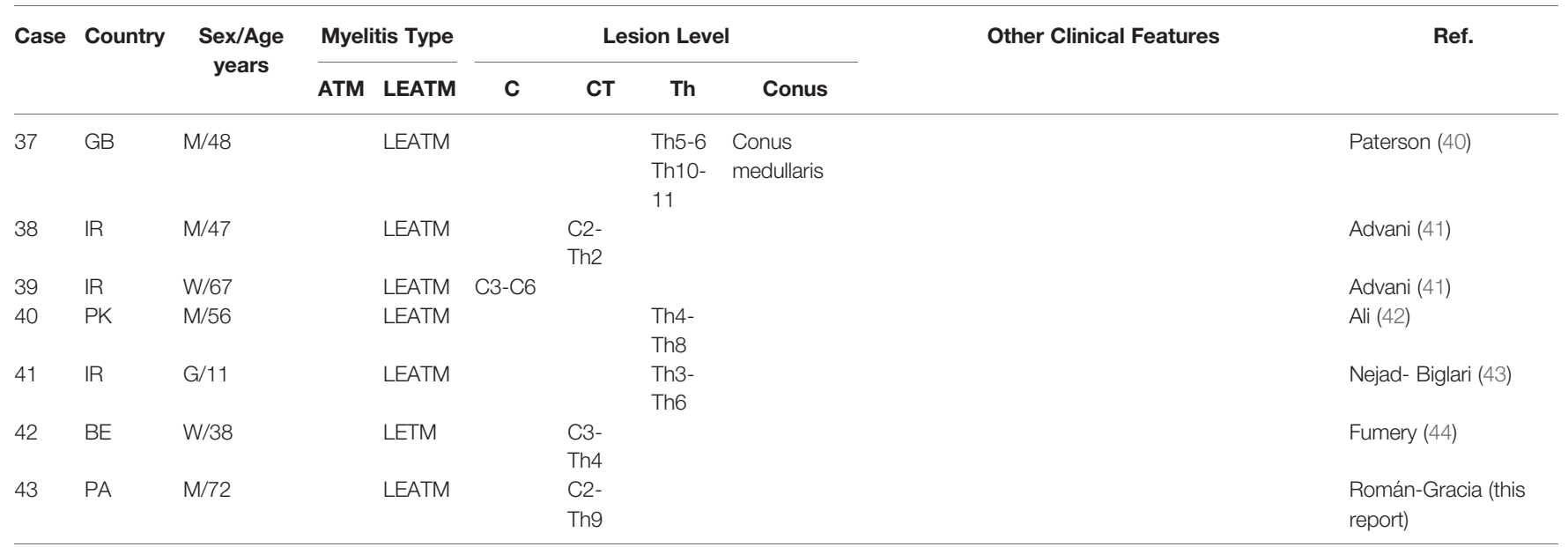

Names of Countries: Australia $=A U$, Belgium $=B E$, Brazil $=B R$, China $=C N$, Denmark $=D K$, Germany $=D E$, India $=I N, I n d o n e s i a=I D, I r a n=I R$, Italy $=I T, M e x i c o=M X, M o l d o v a=M D$, Panama $=P A$, Pakistan $=P K$, Qatar $=Q A$, Spain $=E S$, Switzerland $=C H$, Turkey $=T R$, United Arab Emirates $=A E$, United Kingdom $=$ GB, United States of America $=$ US. ADEM, acute disseminated encephalomyelitis; AMAN, acute motor axonal neuropathy; ANA, antinuclear antibodies; AQP4, aquaporin-4; ATM, acute transverse myelitis; (C, cervical; CSF, cerebrospinal fluid; G, girl; HIV, human immunodeficiency virus; LEATM, longitudinally-extensive acute transverse myelitis; M, man; MOG-IgG, myelin oligodendrocyte glycoprotein antibody-immune globulin G; NMOSD, neuromyelitis optica spectrum disorder; SARS-CoV-2, severe acute respiratory syndrome-coronavirus-2, Th, thoracic; W, woman.

complement C3, C4. Rheumatology consult found no underlying disease. Aquaporin-4 antibody (anti-AQP4) and myelin oligodendrocyte glycoprotein antibody IgG (anti-MOG-IgG) in serum were both negative (Quest diagnostics Nichols Institute, CA).

Gadolinium-enhanced magnetic resonance imaging (MRI) of the brain was normal. MRI of the spinal cord revealed mild cervical and thoracic cord enlargement and swelling with diffuse hyperintensities. On the axial projections, cord hyperintensities at $\mathrm{C}_{4}-\mathrm{C}_{5}$ and $\mathrm{Th}_{3}-\mathrm{Th}_{4}$ were observed with irregular patchy imaging but without contrast enhancement consistent with ATM (Figure 1). No apparent hemorrhagic components were present, and the conus medullaris had normal appearance.

Cerebrospinal fluid (CSF) showed no cells, hyperproteinorraquia of $76 \mathrm{mg} / \mathrm{dl}$ and normal glucose. Meningitis CSF panel was negative for bacteria, yeast and viruses. Gram, acid-fast bacilli and fungus stains were negative. CSF oligoclonal bands (IgG) demonstrated 3 well-defined gamma restriction bands that were not present in serum (Quest diagnostics Nichols Institute, CA).

The patient was treated with a pulse dose of IV methylprednisolone $1 \mathrm{~g} / \mathrm{d}$ for 5 days, enoxaparin $40 \mathrm{mg}$ daily, followed by IV gamma-globulin (IVIG) $30 \mathrm{~g} /$ day for five days. Oral prednisone was prescribed for the next 30 days. He recovered partial strength in his upper limbs $(4+/ 5)$ but the severe spastic paraplegia $(1+/ 5)$ and the neurogenic bladder remained unchanged. He is undergoing physical therapy and rehabilitation treatment.

\section{CASE-FINDING REVIEW}

We performed a comprehensive search of the literature using PubMed, Medline, Scopus, Web of Science, EMBASE, and Google Scholar up to January 5, 2021. For PubMed we used the following key search terms: ("Myelitis, Transverse" [MeSH] OR "Myelitis" [All Fields] OR "Myelitis, Acute" [All Fields] OR "Encephalomyelitis" [All Fields] OR "Neuromyelitis Optica" [MeSH] OR "Myeloneuropathy" [All Fields] OR "Encephalomyelitis, Acute Disseminated" [MeSH] OR "Acute Disseminated Encephalomyelitis” [All Fields]) AND (“COVID19" [MeSH Term] OR "SARS-CoV-2" [MeSH Term] OR "coronavirus" [All Fields]). Table 1 and Supplementary Table 1A list the total 43 patients reported in 21 countries worldwide, as follows: 7 cases from Iran (IR), 6 each from Italy (IT) and the United States of America (US), 4 from the United Kingdom (GB), 2 cases each from Brazil (BR), Spain (ES), and Turkey (TR), plus single case reports from Australia (AU), Belgium (BE), China (CN), Denmark (DK), Germany (DE), India (IN), Indonesia (ID), Mexico (MX), Moldova (MD), Panama (PA), Pakistan (PK), Qatar (QA), Switzerland $(\mathrm{CH})$, and the United Arab Emirates (AE). Patient 10 from the US was published twice $(15,16)$.

\section{RESULTS}

Early reports of neurological complications of COVID-19 from China (2) and France (3) included no cases of ATM. Therefore, it was unexpected to collect 43 cases of COVID-19-associated myelitis in a period of 10 months around the world. Given a total of 86 million COVID-19 cases as of 5 January 2021 (coronavirus.jhu.edu) the incidence of myelitis is 0.5 per million. Based on a single hospital COVID-19 series with 1760 patients from Italy (34), SARS-CoV-2-associated ATM may represent $1.2 \%$ of all neurological complications of COVID-19.

COVID-19-associated ATM was reported in 23 males (53\%) and 20 females (47\%) ranging in age from 21 to 73 years (mean age 49 years) excluding children. There were three age groups: (i) 


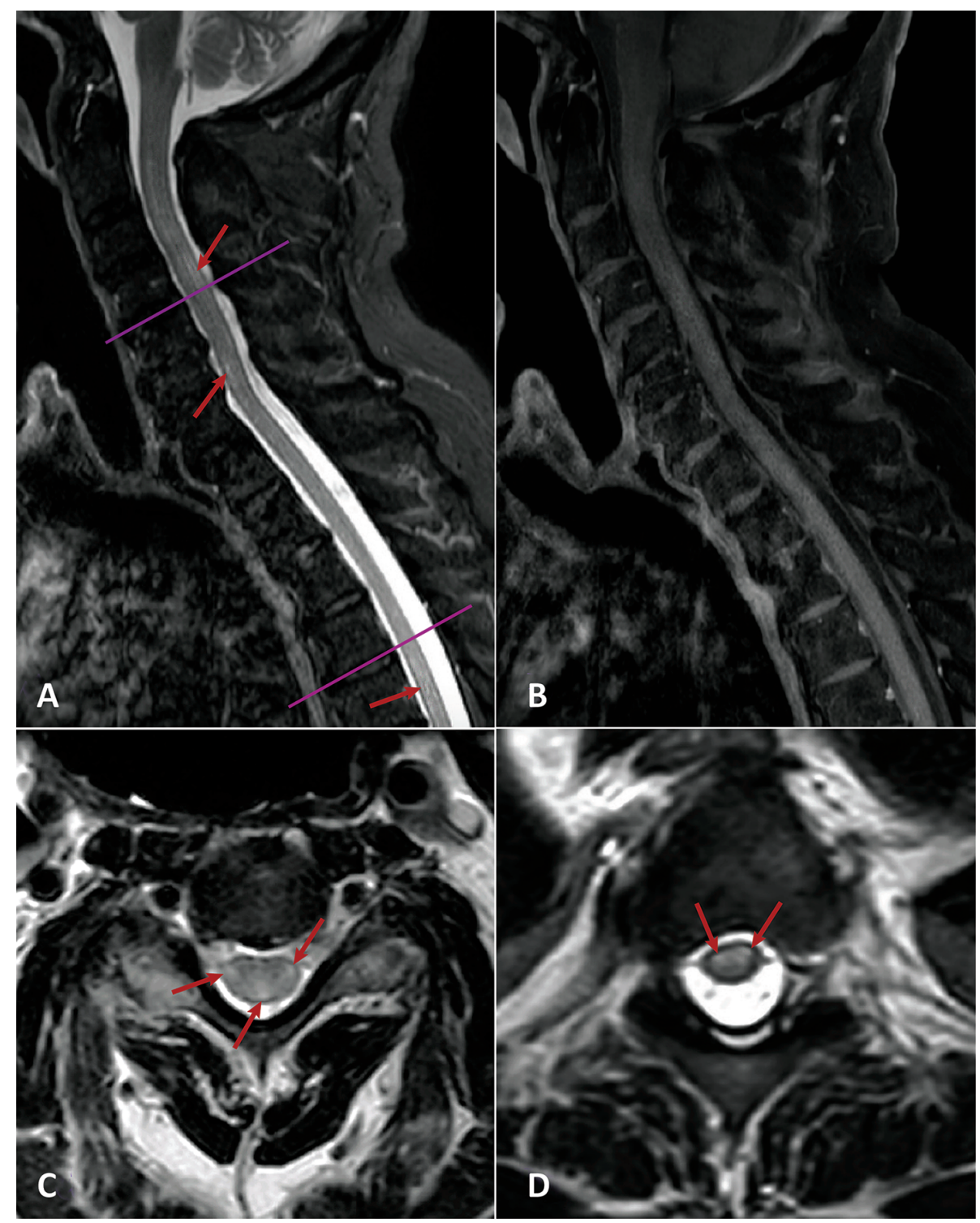

FIGURE 1 Spinal cord MRI. (A) Sagittal Short-T1 Inversion Recovery: Mild cervical cord thickening and diffuse hyperintensities in cervical and dorsal cord (red arrows). (B) Sagittal T1 + gadolinium. No contrast enhancement. (C, D) Axial T2 (C4-5 and T3-4 levels) showing diffuse cord hyperintensities (red arrows).

Pediatric cases: Patient 21, a 3-year-old Navajo girl in the USA (27), Patient 31, a 14-year-old girl in Turkey (36), and Patient 41, an 11-year-old girl from Iran (43). (ii) Young adult cases: 13 patients, 7 men and 6 women, ages 21-42 years with mean age of 29 years. (iii): Older adults: 27 patients, 18 men and 9 women, ranging from $44-73$ years (mean age 58 years).

The main manifestations of the spinal cord lesions based on clinical examination included two major groups, quadriplegia and paraplegia. There were $27 / 40$ patients (58\%) with tetraparesis/quadriplegia resulting from cervical cord-upper thoracic cord lesions compared with 15/40 (42\%) with acute paraparesis/paraplegia from thoracic cord lesions. The anatomical distribution of the spinal cord lesions by MRI imaging was reported in 40 cases (Table 1 and
Supplementary Table 1A). Localized ATM lesions affected $\leq 3$ cord segments in 12 cases $(30 \%)$ at cervical (5 cases) and thoracic cord (7 cases) levels, and 28 cases $(70 \%)$ had longitudinally-extensive ATM (LEATM) involving $\geq 4$ spinal cord segments in cervicothoracic (18 cases) and thoracolumbar-sacral regions (10 cases). In 3 case reports the lesions are described as 'myelitis' without further clinical information. Cervical cord lesions extended in some cases to the brainstem causing rhombencephalitis (8), as well as involvement of pons and medulla oblongata. Patient 4 from Denmark (10) had the most extensive lesions reported affecting the entire spinal cord from the medulla oblongata to the conus medullaris. Patients 18, 36 and 37 had lesions affecting the conus medullaris. 
The latency period from the onset of COVID-19 symptoms to the first neurological manifestations followed a dual distribution: (i) Short latency: 15 hours to 5 days in 11/34 patients (32\%) and (ii) Long latency: 10 days to 6 weeks in 68\% (Table 1 and Supplementary Table 1A). The shorter latency period may indicate a direct neurotropic effect of SARS-CoV2 during the initial infection causing para-infectious myelitis. Longer latency periods may indicate a post-infectious neurological complication resulting from the host response to the virus. No particular geographic origin, distribution by sex or age group, nor clinical picture were associated with shorter or longer latency periods. Treatments included steroids in most patients, along with IVIg in a few instances; some patients also received respiratory support. There were 2 deaths reported (Patient 17 from Qatar and Patient 23 from India). SARS-CoV-2 RNA PCR in the CSF was positive in 2 cases $(18,24)$.

\section{ATM AND OTHER NEUROINFLAMMATORY SYNDROMES}

According to Hartung and Aktas (47) a number of neuroimmunological disorders affecting CNS and PNS are expected to occur during COVID-19. Although the immune mechanisms causing ATM remain unknown other neurological disorders of neuroimmune nature were reported concurrently with the myelitis (Table 1 and Supplementary Table 1A). These included acute disseminated encephalomyelitis (ADEM), neuromyelitis optica (NMO) and acute motor axonal neuropathy (AMAN).

\section{ACUTE DISSEMINATED ENCEPHALOMYELITIS (ADEM)}

Reichard et al. (48) reported the neuropathological findings, extensive vascular lesions and perivenous demyelination of ADEM in association with COVID-19. Myelitis as part of ADEM was diagnosed in $8 / 40$ patients $(20 \%)$ summarized in Table 1 and Supplementary Table 1A. In contrast with the overall male preponderance in this series, ADEM with ATM affected predominantly women (67\%) ranging in age from 27 64 years (mean age 43 years). Lesions revealed by spinal cord MRI included LEATM from medulla oblongata and cervicothoracic cord $\left(\mathrm{Th}_{6}\right)$ in Patient 3 (8); cervicothoracic spinal cord lesions down to the conus medullaris in Patient 18 (24); $\mathrm{C}_{1-7}-\mathrm{Th}_{2}$ in Patient 35 (40); and, $\mathrm{Th}_{5-6}$ and $\mathrm{Th}_{10-11}$ down to the conus medullaris in Patient 37 (40). ATM at $\mathrm{Th}_{8}$ level occurred in Patient 8 (13); at $\mathrm{C}_{2-3}$ level in Patient 16 (22); pons and medulla-cord junction in Patient 19 (25); and, intramedullary lesion of the conus medullaris in Patient 36 (40). Brain MRI lesions consistent with ADEM included among others, multiple T1 post-Gd enhancing white matter lesions plus bilateral edema of the optic nerves; hyperintense
FLAIR lesions in the medial temporal lobe; bilateral lesions involving cerebral white matter, corpus callosum and brainstem including pons and medulla-cord junction.

\section{NEUROMYELITIS OPTICA (NMO) AND NMO SPECTRUM DISORDERS (NMOSD)}

NMO and NMOSD are relatively common conditions in neuroimmunology (49-51) previously reported after SARSCoV-2 infection (52). We found 3 patients with myelitis and visual loss due to optic nerve edema diagnosed with NMO. Patient 8 (13), is a 64-year-old woman with ATM and visual loss. Patient 33 (38), is a 26-year-old Hispanic man from the USA with positive MOG-IgG antibodies who developed papilledema, blindness and dysesthesias of the upper extremities due to bilateral optic neuritis and LEATM cord lesions from $\mathrm{C}_{4}-\mathrm{Th}_{2}$. Patient 25, from Rio de Janeiro, Brazil (31) is a 51-year-old Caucasian woman, with a 2 -week history of COVID-19 who developed band-like dysesthesias at the $\mathrm{Th}_{6-10}$ dermatomes, urinary retention, leg numbness and paraparesis. Brain MRI showed enhancing T2/FLAIR lesions in anterior fornix and subfornical organ. Spinal cord MRI demonstrated LEATM at Th6-10 with lumbar radiculitis. Serum ANA was positive (1:320). Anti-AQP4 antibodies were positive in serum and CSF. This encephalomyeloradiculitis is probably a form of COVID-19associated NMOSD. According to Jarius et al. (53) NMO and NMOSD are caused in $>80 \%$ of cases by pathogenetic IgG autoantibodies to AQP4 but only 1 case was positive in this cohort. A 15-year-old Caucasian boy with SARS-CoV-2associated NMO reported by de Ruijter at al (52). had blindness without myelitis but with positive anti-MOGIgG antibodies.

\section{ACUTE MOTOR AXONAL NEUROPATHY (AMAN)}

AMAN may be found in patients with clinical Guillain-Barré syndrome (GBS), an acute immune-mediated polyradiculoneuropathy reported as the most common form of peripheral nerve lesion in patients with $\operatorname{COVID}-19(54,55)$. Based on electrophysiological features GBS can be classified into several subtypes including acute inflammatory demyelinating polyneuropathy (AIDP), acute motor and sensory axonal neuropathy (AMSAN), and AMAN $(54,56)$.

We found reports of 2 patients that presented concurrently AMAN with ATM indicating simultaneous involvement of CNS and PNS. Patient 10, a 61-year-old woman from the US $(15,16)$ developed quadriparesis due to LEATM affecting $\mathrm{C}_{1^{-}}$ $\mathrm{Th}_{1}$ and concurrent AMAN with negative anti-MOG-IgG and anti-AQP4-IgG antibodies. Patient 26, a 70-year-old woman from Italy (32) developed quadriparesis from ATM at $\mathrm{C}_{7}-\mathrm{Th}_{1}$ and AMAN with positive anti-GD1b IgM antibodies. 


\section{OTHER IMMUNE/INFLAMMATORY FACTORS}

In addition to the above patients other immune or inflammatory mechanisms may have contributed to COVID-19-associated ATM. Patient 22 is a 27-year-old HIV-positive man from Moldova (28) who developed paraplegia due to LEATM involving $\mathrm{C}_{4}-\mathrm{Th}_{5}$. Patient 32 is a 72-year-old man from Mexico (37) with preexisting cervical spondylotic myelopathy that evolved to tetraparesis after SARSCoV-2 infection due to ATM at the $\mathrm{C}_{1}-\mathrm{C}_{3}-\mathrm{C}_{6}$ levels.

\section{DISCUSSION}

The neurotropism of the coronaviruses in general and SARS-CoV-2 in particular has been well demonstrated (57-60). Moreover, the numerous neurological complications of COVID-19 are well recognized $(1-4,34,40,47,48,54,55,57,61)$. Symptoms reflecting central nervous system involvement include headache, anosmia and dysgeusia, agitation, delirium, and impaired consciousness $(1,61)$. Stroke is common, probably reflecting the endoteliitis (62) and small-vessel brain lesions (4) causing brain hemorrhages, arterial and venous thromboses, and subarachnoid hemorrhage, as well as rare cases of acute hemorrhagic necrotizing encephalopathy $(48,61)$. Neuropathological examination of fatal adult cases of COVID-19 (63-65) showed in addition to vascular lesions (4) low-grade localized encephalitis affecting brainstem respiratory and cardiovascular centers (63).

According to Paterson et al. (40) the postulated mechanisms causing ATM and the various neurological syndromes associated with SARS-CoV-2 include, either individually or in combination, direct viral neuronal injury (57-60) and the host's secondary hyperinflammation syndrome $(61,66,67)$. SARS-CoV-2 enables interleukin (IL)-1 synthesis and release (68) leading to inflammasome activation. Also, IL-6, a proinflammatory mediator, is elevated in COVID-19 and induces CNS immune responses (68). Type I interferon (IFN) is dysregulated in COVID-19 and can affect innate and acquired immunity (69). COVID-19 patients exhibit increased circulating levels of IL-2, IL-8, IL-17, granulocyte colony-stimulating factor, granulocytemacrophage colony-stimulating factor, interferon gammainduced protein 10 , and monocyte chemoattractant protein 1 $(69,70)$. IFN release can result in inflammation and immune system suppression (70). These immune factors may lead to the so-called "cytokine storm" syndrome that triggers coagulopathy and thrombosis (71). Also, of critical importance during COVID-19 are the para- and post-infectious inflammatory or immune-mediated neurological disorders (72-75), also observed after vaccination $(76,77)$, that affect both the CNS and the PNS causing GBS, ADEM, NMOSD, and ATM, among others.

\section{ACUTE TRANSVERSE MYELITIS (ATM)}

\section{Definition and Differential Diagnosis}

The term ATM is used here to identify patients with myelitis described during COVID-19. Most COVID-19-associated ATM cases reported here fulfill the strict definition of the Transverse Myelitis Consortium Working Group (78) requiring clinical evidence of bilateral sensory, motor, or autonomic dysfunction referable to the spinal cord, and confirmed by MRI images.

ATM is different from acute flaccid myelitis or AFM (79) the predominantly pediatric form of acute flaccid paralysis with anterior myelitis or "polio-like syndrome" with spinal cord gray matter lesions. Epidemiological data for pediatric cases of AFM from a national surveillance program in the US (80) reported as of July 2020 a total of 633 cases of AFM with a median age of 5.3 years with peaks in 2014, 2016 and 2018. Nonpolio enteroviruses, including EV-D68 and EV-A71, are the most frequent etiological agents (81). Children are relatively unaffected by SARS-CoV-2, probably because of low ACE2 receptors in the olfactory mucosa (82) and there has been no increase in cases of AFM up to July 2020. Patient 21 (27), a 3 -year-old Navajo girl, is the youngest pediatric case of SARS$\mathrm{CoV}$-2-associated ATM reported in this series. Three weeks after an asymptomatic COVID-19 infection she developed a flaccid quadriparesis as a result of LEATM extending from the lower medulla and $\mathrm{C}_{1}$ to the $\mathrm{Th}_{6}$ spinal cord segments. These lesions are clearly different from those of pediatric AFM (83).

According to West and colleagues (84), ATM remains a rare immune-mediated neurological condition with an estimated incidence of up to 3 per 100,000 patient years (0.003\%). ATM can be caused by autoimmune, inflammatory, and infectious agents but the main differential diagnosis is with multiple sclerosis. Clinical features and imaging usually eliminate from the differential diagnosis of ATM other noninflammatory conditions such as traumatic, compressive, neoplastic or vascular lesions (84).

\section{Pathogenesis of COVID-19-Associated ATM}

The latency period between SARS-CoV-2 infection and onset of the neurological symptoms was unknown in many instances because of asymptomatic COVID-19. In most cases (68\%) the latency period ranged from 10 days to 6 weeks; in the remaining $32 \%$ ( $11 / 34$ cases) the latency period ranged from hours to 5 days; the shortest period was 15 hours for Patient 22, the HIVpositive man from Moldova (28). Very short latency periods of respectively 2 and 3 days occurred for Patient 11 from UAE (17) and Patient 17 from Qatar (23). It is unknown if these two patients had been previously infected with the Middle East Respiratory Syndrome coronavirus (MERS-CoV).

The neurological complications of viral infections can be either para-infectious, i.e., due to direct viral neurotropism, or post-infectious, i.e., resulting from immune-mediated reactions against the virus $(48,57-59,61-68)$. Except for the three parainfectious cases mentioned above, most cases of SARS-CoV-2associated myelitis had longer latency periods suggesting a postinfectious origin.

According to Blackburn and Wang (72), the proposed mechanisms of post-infectious neurological disorders include molecular mimicry, epitope spreading, bystander activation and polyclonal B-cell activation. Molecular mimicry is due to the presence in microorganisms of epitopes that share marked similarity in peptide sequence or three-dimensional structure 
to host's antigens. Therefore, lymphocytes activated by the infection may cross-react with self-antigens. In epitope spreading the specific initial response to an antigen is broadened to include other different epitopes. Also, during the immune response to a highly virulent pathogen autoreactive lymphocytes may be activated during the inflammatory cascade resulting in autoimmunity by "bystander activation." Finally, polyclonal B-cell activation may occur with chronic viral infections that persist in the host such as herpesviruses. Molecular mimicry and bystander activation appear to be the most likely mechanisms explaining SARS-CoV-2-associated ATM. The antibodies reported in patients with COVID-19associated ATM included 3 cases of positive anti-MOG-IgG antibodies and single cases of positive anti-GD1b IgM antibodies, ANA, and anti-AQP4 antibodies in serum and CSF.

\section{POST-VACCINATION ATM}

Neurological complications of vaccination were first recognized in 1885 with Pasteur's rabies vaccine obtained from rabbits' spinal cords. More recently, in 1977, we reported 21 cases of GBS and brain demyelination (77) resulting from the use in Colombia of the suckling mouse brain (SMB) rabies vaccine containing neural tissue antigens causing neurological complications that included GBS, ADEM, chronic leukoencephalitis, and myelitis (77). Concurrent involvement of CNS and PNS occurs in postviral infections such as Zika (75). These lesions resemble those of experimental autoimmune encephalomyelitis induced in animals with the use of myelin antigens and Freund's adjuvant $(74,76$, 84 ). Current rabies vaccine obtained from tissue culture of human diploid cells eliminated this problem.

The US national vaccination campaign in 1976 against the A New Jersey "swine flu" influenza using the A/NJ/1976/H1N1 vaccine was associated with increased incidence of GBS (85). Nachamkin et al. (86) postulated that Campylobacter jejuni antigens that mimic human gangliosides capable of inducing an anti-GM $\mathrm{GM}_{1}$ antibody response could have caused GBS. Campylobacter antigens were not present in any of the vaccines examined. However, these authors demonstrated that the 1976, 1991-1992, 2004-2005 influenza vaccines induced IgG and IgM anti-GM $\mathrm{GM}_{1}$ antibodies in mice. Recent cases of ATM following $\mathrm{H} 1 \mathrm{~N} 1$ vaccination have been reported (87-90) indicating that influenza vaccines may induce immune mechanisms targeting the spinal cord. It may be important to notice that the COVID-19 ChAdOx1 nCoV-1 vaccine (AZD1222) contains chimpanzee adenovirus antigens as adjuvant.

In 2003, an experimental vaccine (AN1792) containing synthetic aggregated A 342 fragments with QS-21 as adjuvant targeting the amyloid precursor protein with the aim of preventing the development of Alzheimer's disease resulted in meningoencephalitis in $6 \%$ of the vaccinated patients (91). The trial was discontinued and neuropathology studies (92) confirmed the presence of meningoencephalitis with strong $\mathrm{MCH}$ class I immunoreactivity in collapsed amyloid plaques and multinucleated giant cells suggesting that a fragment of $\beta$ amyloid was the possible origin of the post-vaccination reaction.

Numerous other vaccines have caused neurological postvaccination complications including diphtheria-tetanus-polio, measles, mumps, rubella, Japanese B encephalitis, and pertussis. According to Karussis and Petrou (76), the most recent cases of CNS demyelination after vaccination include vaccines against influenza, human papilloma virus, hepatitis $\mathrm{A}$ or B, measles, rubella, yellow fever, anthrax, meningococcus and tetanus. Other than GBS, ATM, and ADEM, other postvaccination reactions include neuromyelitis optica (NMOSD), isolated ophthalmoplegia, brachial neuritis and other mononeuropathies. Post-vaccination reactions have declined with the use of recombinant proteins, rather than in vivo infected animal tissue (73). Recent research on the immunopathogenesis of ATM (93) has emphasized the role of interleukins IL-6 and IL-17. In myelitis, IL-6 is elevated in the CSF and predicts disability (94). Production of both IL-6 and IL17 by peripheral blood mononuclear cells is increased in ATM (94). IL-17 regulates cytokines (TNF $\alpha$, IL-1 $\beta$ and IL-6) to stimulate IL- 6 production by astrocytes. The role of adjuvants as contributing factors to the immune and inflammatory reactions to vaccines has also been emphasized (95).

\section{ChAdOx1 nCoV-19 Vaccine (AZD1222) Trials}

The ChAdOx1 nCoV-19 vaccine (AZD1222) consists of a replication-deficient chimpanzee adenoviral ChAdOx1 containing the SARS-CoV-2 structural surface vector glycoprotein antigen (spike protein; $\mathrm{nCoV}-19$ ) gene (45). The safety and efficacy report of four randomized controlled trials conducted in Brazil, South Africa and Great Britain for the AZD1222 COVID-19 vaccine informed the occurrence of three cases of ATM as serious adverse events $(45,46)$.

One participant developed ATM 14 days after ChAdOx1 $\mathrm{nCoV}-19$ booster vaccination and was diagnosed as idiopathic, short segment, spinal cord demyelination possibly related to vaccination $(45,46)$. The second participant developed ATM 10 days after a first vaccination with ChAdOx1 $\mathrm{nCoV}-19$. It was initially assessed as possibly vaccine-related but later considered unlikely when further investigation revealed pre-existing, but previously unrecognised, multiple sclerosis $(45,46)$. The third patient with ATM occurred in a control subject 68 days after receiving the meningococcal conjugate (MenACWY) vaccine. Initially considered possibly related, it was finally considered unlikely to be vaccine-related by neurological experts $(45,46)$. However, no information was provided regarding COVID-19 infection in this unvaccinated subject. These three ATM serious adverse events resulted in temporarily halting of the vaccine trial until the affected participants began to show signs of recovery.

The occurrence of three reported ATM cases among 11,636 participating subjects is extremely high considering the worldwide incidence of $0.5 /$ million COVID-19-associated ATM cases found in this report during year 1 of the pandemic. Moreover, Agmon-Levin et al. (96) in a systematic review (19702009) fund in 39 years only 37 reported cases of ATM in association with several different vaccines. 


\section{CONCLUSION}

This review confirms that ATM is not uncommon as a neurological complication associated with COVID-19 infection around the world, responsible perhaps for $1.2 \%$ of all neurological complications caused by this coronavirus. It occurs acutely in a small number of patients as a parainfectious manifestation but most cases of SARS-CoV-2associated ATM have longer latency periods suggesting a postinfectious origin. These facts suggest probable viral antigen(s) in SARS-CoV-2 target the spinal cord -perhaps also present in the COVID-19 vaccine AZD1222 or its chimpanzee adenovirus adjuvant- and may induce immune mechanisms leading to ATM. Research to identify the responsible antigen(s) and the immunopathogenesis of COVID-19-associated ATM must be encouraged.

\section{DATA AVAILABILITY STATEMENT}

The datasets presented in this article are not readily available. Requests to access the datasets should be directed to gcroman@houstonmethodist.org.

\section{REFERENCES}

1. Román GC, Spencer PS, Reis J, Bouguet A, El Alaoui Faris M, Katrak SM, et al. on behalf of the WFN Environmental Neurology Specialty Group. The neurology of COVID-19 revisited: A proposal from the Environmental Neurology Specialty Group of the World Federation of Neurology to implement international neurological registries. J Neurol Sci (2020) 414:116884. doi: 10.1016/j.jns.2020.116884

2. Mao L, Jin H, Wang M, Hu Y, Chen S, He Q, et al. Neurologic manifestations of hospitalized patients with coronavirus disease 2019 in Wuhan, China. JAMA Neurol (2020) 77(6):683-90. doi: 10.1001/jamaneurol.2020.1127

3. Helms J, Kremer S, Merdji H, Clere-Jehl R, Schenck M, Kummerlen C, et al. Neurological features in severe SARS-CoV-2 infection. N Engl J Med (2020) 382:2268-70. doi: 10.1056/NEJMc2008597

4. Lee M-H, Perl DP, Nair G, Li W, Maric D, Murray H, et al. Microvascular injury in the brains of patients with Covid-19. N Engl J Med (2020) 383:481-3. doi: 10.1056/NEJMc2033369

5. Borchers AT, Gershwin ME. Transverse myelitis. Autoimmun Rev (2012) 11:231-48. doi: 10.1016/j.autrev.2011.05.018

6. Zhao K, Huang J, Dai D, Feng Y, Liu L, Nie S. Acute myelitis after SARS-CoV2 infection: A case report. medRxiv (2020) Preprint. doi: 10.1101/ 2020.03.16.20035105

7. Saberi A, Ghayeghran A, Hatamian H, Hosseini-Nejad M, Bakhshayesh Eghbali B. COVID-19- associated myelitis, para/post infectious or infectious myelitis. Caspian J Neurol Sci (2020) 6:132-8. doi: 10.32598/ CJNS.6.21.1

8. Wong PF, Craik S, Newman P, Makan A, Srinivasan K, Crawford E, et al. Lessons of the month 1: A case of rhombencephalitis as a rare complication of acute COVID-19. Clin Med (2020) 20:293-9. doi: 10.7861/clinmed.2020-0182

9. Zanin L, Saraceno G, Panciani PP, Renisi G, Signorini L, Migliorati K, et al. SARS- CoV-2 can induce brain and spine demyelinating lesions. Acta Neurochir (Wien) (2020) 162:1491-4. doi: 10.1007/s00701-020-04374-x

10. Sarma D, Bilello LA. A case report of acute transverse myelitis following novel coronavirus infection. Clin Pract cases Emerg Med (2020) 4:321-3. doi: 10.5811/cpcem.2020.5.47937

11. Sotoca J, Rodríguez-Álvarez Y. COVID-19-associated necrotizing myelitis. Neurol Neuroimmunol Neuroinflamm (2020) 7:e803. doi: 10.1212/ NXI.0000000000000803

\section{AUTHOR CONTRIBUTIONS}

GR and FG drafted the manuscript. FG, AT, AP, KG, and DH provided clinical data. GR, FG, and KG participated in the search of the literature. All authors contributed to the article and approved the submitted version.

\section{FUNDING}

Prof. Román's research is funded by the Blanton Endowed Chair, the Wareing Family Research Fund and the David Cabello Research Fund at Houston Methodist Hospital.

\section{SUPPLEMENTARY MATERIAL}

The Supplementary Material for this article can be found online at: https://www.frontiersin.org/articles/10.3389/fimmu.2021. 653786/full\#supplementary-material

Supplementary Table 1A | Clinical and MRI Data of Published Cases of SARSCoV-2-Associated Myelitis (March-2020 January-2021).

12. Munz M, Wessendorf S, Koretsis G, Tewald F, Baegi R, Krämer S, et al. Acute transverse myelitis after COVID-19 pneumonia. J Neurol (2020) 267:2196-7. doi:10.1007/s00415-020-09934-w

13. Novi G, Rossi T, Pedemonte E, Saitta L, Rolla L, Roccatagliata L, et al. Acute disseminated encephyalomyelitis after SARS-CoV-2 infection. Neurol Neuroimmunol Neuroinflamm (2020) 7:e797. doi: 10.1212/NXI.00000 00000000797

14. Giorgianni A, Vinacci G, Agosti E, Cariddi LP, Mauri M, Baruzzi F, et al. Transient acute-onset tetraparesis in a COVID-19 patient. Spinal Cord (2020) 58:1042-4. doi: 10.1038/s41393-020-0493-8

15. Valiuddin H, Skwirsk B, Paz-Arabo P. Acute transverse myelitis associated with SARS-CoV-2: A case report. Brain Behav Immun - Health (2020) 5:100091. doi: 10.1016/j.bbih.2020.100091

16. Maideniuc $\mathrm{C}$, Memon AB. Acute necrotizing myelitis and acute motor axonal neuropathy in a COVID-19 patient. J Neurol (2020) 268:739. doi: 10.1007/ s00415-020-10145-6

17. Al Ketbi R, Al Nuaimi D, Al Mulla M, Al Talai N, Samir M, Kumar N, et al. Acute myelitis as a neurological complication of COVID-19: A case report and MRI findings. Radiol Case Rep (2020) 15:1591-5. doi: 10.1016/ j.radcr.2020.06.001

18. Domingues RB, Mendes-Correa MC, Vilela de Moura Leite FB, Cerdeira Sabino E, Zanotti Salarini D, Claro I, et al. First case of SARS-CoV-2 sequencing in cerebrospinal fluid of a patient with suspected demyelinating disease. J Neurol (2020) 267:3154-6. doi: 10.1007/s00415-020-09996-w

19. Zoghi A, Ramezani M, Roozbeh M, Darazam IA, Sahraian MA. A case of possible atypical demyelinating event of the central nervous system following COVID-19. Multiple Sclerosis Related Dis (2020) 44:102324. doi: 10.1016/ j.msard.2020.102324

20. Durrani M, Kucharski K, Smith Z, Fienc S. Acute transverse myelitis secondary to severe acute respiratory syndrome coronavirus 2 (SARS-CoV2): A case report. Clin Pract cases Emerg Med (2020) 4:344-8. doi: 10.5811/ cpcem.2020.6.48462

21. Zachariadis A, Tulbu A, Strambo D, Dumoulin A, Di Virgilio G. Transverse myelitis related to COVID-19 infection. J Neurol (2020) 267:3459-61. doi: 10.1007/s00415-020-09997-9

22. Otluoglu GD, Yener U, Demir MK, Yilmaz B. Encephalomyelitis associated with COVID-19 infection: Case report. Br J Neurosurg (2020) 1-3. doi: 10.1080/02688697.2020.1787342 
23. Abdelhady M, Elsotouhy A, Vattoth S. Acute flaccid myelitis in COVID-19. BJR Case Rep (2020) 6:20200098. doi: 10.1259/bjrcr.20200098

24. Utukuri PS, Bautista A, Lignelli A, Moonis G. Possible acute disseminated encephalomyelitis related to severe acute respiratory syndrome coronavirus 2 infection. AJNR Am J Neuroradiol (2020) 41:E82-3. doi: 10.3174/ajnr.A6714

25. McCuddy M, Kelkar P, Zhao Y, Wicklund D. Acute demyelinating encephalomyelitis (ADEM) in COVID-19 infection: A case series. medRxiv (2020) Preprint. doi: 10.1101/2020.07.15.20126730

26. Chow CCN, Magnussen J, Ip J, Su Y. Acute transverse myelitis in COVID-19 infection. BMJ Case Rep (2020) 13:e236720. doi: 10.1136/bcr-2020-236720

27. Kaur H, Mason JA, Bajracharya M, McGee J, Gunderson MD, Hart BL, et al. Transverse myelitis in a child with COVID-19. Pediatr Neurol (2020) 112:5-6. doi: 10.1016/j.pediatrneurol.2020.07.017

28. Lisnic V, Nemtan V, Hacina E, Topciu G, Manole E, Thumher MM, et al. Acute transverse myelitis in a HIV-positive patient with COVID-19. Res Square (2020) Preprint. doi:10.21203/rs.3.rs-50901/v1

29. Chakraborty U, Chandra A, Ray AK, Biswas P. COVID-19-associated acute transverse myelitis: A rare entity. BMJ Case Rep (2020) 13:e238668. doi: 10.1136/bcr-2020-238668

30. Hazrati E, Farahani RH, Asl AN, Shahali H. Acute transverse myelitis after SARS-CoV-2 infection: A rare complicated case of rapid onset paraplegia in a male veteran. Res Square (2020) Preprint. doi: 10.21203/rs.3.rs-68798/v1

31. Corrêa DG, de Souza Lima FC, da Cruz Bezerra D, Coutinho ACJr, Hygino da Cruz LC. COVID- 19 associated with encephalomyeloradiculitis and positive anti-aquaporin-4 antibodies: Cause or coincidence? Multiple Sclerosis J (2020) 00(0):1-4. doi: 10.1177/1352458520949988

32. Masuccio FG, Barra M, Claudio G, Claudio S. A rare case of acute motor axonal neuropathy and myelitis related to SARS-CoV-2 infection. J Neurol (2020) 1-4. Online ahead of print. doi:10.1007/s00415-020-10219-5

33. Baghbanian SM, Namazi F. Post COVID-19 longitudinally extensive transverse myelitis (LETM) - A case report. Acta Neurol Belgica (2020) 1-2. Online ahead of print. doi:10.1007/s13760-020-01497-x

34. Rifino N, Censori B, Agazzi E, Alimonti D, Bonito V, Camera G, et al. Neurologic manifestations in 1760 COVID-19 patients admitted to Papa Giovanni XXIII Hospital, Bergamo, Italy. J Neurol (2020) 1-8. Online ahead of print. doi: 10.1007/s00415-020-10251-5

35. Águila-Gordo D, Flores-Barragán JM, Ferragut-Lloret F, LaRosa-Salas B, Porras-Leal L, Villa Guzman JC. Acute myelitis and SARS-CoV-2 infection. A new etiology of myelitis? J Clin Neurosci (2020) 80:280-1. doi: 10.1016/ j.jocn.2020.07.074

36. Güler M, Keskin F, Hüseyin T. Acute myelitis secondary to COVID-19 in an adolescent: Causality or coincidence? New Trend Med Sci (2020) 1:132-6.

37. Guadarrama-Ortiz P, Choreño-Parra JA, Pacheco-Sánchez FJ, Ponce-Sánchez JM, García- Quintero G, Rodríguez-Muñoz PE, et al. Chronic subclinical spondylotic myelopathy exacerbated by COVID-19: A case report. Interdiscip Neurosurg: Adv Tech Case Manage (2021) 23:100896. doi: 10.1016/ j.inat.2020.100896

38. Zhou S, Jones-Lopez EC, Soneji DJ, Azevedo CJ, Patel V. Myelin oligodendrocyte glycoprotein antibody-associated optic neuritis and myelitis in COVID-19. J Neuro Ophthalmol (2020) 00:1-5. doi: 10.1097/ WNO.0000000000001049

39. Munir B, Rianawati SB, Kurnianwan SN, Satoso WM, Arisetijono E, Candradikusuma D, et al. Neurological manifestation on hospitalized patient with probable COVID-19 in Saiful Anwar Hospital in Indonesia (serial cases). Malang Neurol J (2020) 6:51-5. doi: 10.21776/ ub.mnj.2020.006.02.1

40. Paterson RW, Brown RL, Benjamin L, Nortley R, Wiethoff S, Bharucha T, et al. The emerging spectrum of COVID-19 neurology: Clinical, radiological and laboratory findings. Brain (2020) 143:3104-20. doi: 10.1093/brain/ awaa240

41. Advani S, Zali A, Ommi D, Fatemi A, Khoshnoud RJ, Ashrafi F. Transverse myelitis in COVID-19 patients: Report of two cases. Res Square (2020) Preprint. doi:10.21203/rs.3.rs-107744/v1

42. Ali L, Khan A, Elalamy O, Canibano B, Al Hatou M, Ghulam A, et al. A rare presentation of acute flaccid myelitis in COVID-19 patient: A case report. Pakistan J Neurol Sci (2020) 15(3):22-6.

43. Nejad Biglari H, Sinaei R, Pezeshki S, Khajeh Hasani F. Acute transverse myelitis of childhood due to novel coronavirus disease 2019: The first pediatric case report and review of literature. Iran J Child Neurol (2021) 15:107-12. doi: $10.22037 /$ ijcn.v15i1.31579

44. Fumery T, Baudar C, Ossemann M, London F. Longitudinally extensive transverse myelitis following acute COVID-19 infection. Mult Scler Relat Disord (2021) 48:102723. doi: 10.1016/j.msard.2020.102723

45. Voysey M, Clemens SAC, Madhi SA, Weckx LY, Folegatti PM, Aley PK, et al. Safety and efficacy of the ChAdOx1 nCoV-19 vaccine (AZD1222) against SARS-CoV-2: An interim analysis of four randomised controlled trials in Brazil, South Africa, and the UK. Lancet (2021) 397:99-111. doi:10.1016/ S0140-6736(20)32661-1

46. Knoll MD, Wonodi C. Oxford-AstraZeneca COVID-19 vaccine efficacy. Lancet (2021) 397:72-4. doi: 10.1016/S0140-6736(20)32623-4

47. Hartung H-P, Aktas O. COVID-19 and management of neuroimmunological disorders. Nat Rev Neurol (2020) 16:347-8. doi: 10.1038/s41582-020-0368-9

48. Reichard RR, Kashani KB, Boire NA, Constantopoulos E, Gao Y, Luchinett C. Neuropathology of COVID-19: A spectrum of vascular and acute disseminated encephalomyelitis (ADEM)-like pathology. Acta Neuropathol (2020) 140:1-6. doi: 10.1007/s00401-020-02166-2

49. Kleiter I, Gahlen A, Borisow N, Fischer K, Wernecke K-D, Wegner B, et al. on behalf of the Neuromyelitis Optica Study Group. Neuromyelitis Optica: Evaluation of 871 attacks and 1,153 treatment courses. Ann Neurol (2016) 79:206-16. doi: 10.1002/ana.24554

50. Hartung H-P, Aktas O. Old and new breakthroughs in neuromyelitis optica. Lancet (2020) 20:280-1. doi: 10.1016/S1474-4422(20)30062-4

51. Borisow N, Mori M, Kuwabara S, Friedemann P. Diagnosis and treatment of NMO Spectrum Disorder and MOG-Encephalomyelitis. Front Neurol (2018) 9:888. doi: 10.3389/fneurol.2018.00888

52. de Ruijter NS, Kramer G, Gons RAR, Hengstman GJD. Neuromyelitis optica spectrum disorder after presumed COVID-19 infection: A case report. Mult Scler Relat Disord (2020) 46:102474. doi: 10.1016/j.msard.2020.102474

53. Jarius S, Paul F, Weinshenker BG, Levy M, Kim HJ, Wildemann B, et al. Neuromyelitis optica. Nat Rev Dis Primers (2020) 6:85. doi: 10.1038/s41572020-0214-9

54. Andalib S, Biller J, Di Napoli M, Moghimi N, McCullough LD, Rubinos CA, et al. Peripheral nervous system manifestations associated with COVID-19. Curr Neurol Neurosci Rep (2021) 21:9. doi: 10.1007/s11910-021-01102-5

55. Toscano G, Palmerini F, Ravaglia S, Ruiz L, Invernizzi P, Cuzzoni MG, et al. Guillain-Barré syndrome associated with SARS-CoV-2. N Engl J Med (2020) 382:2574-6. doi: 10.1056/NEJMc2009191

56. Kaida K. Guillain-Barré syndrome. Adv Exp Med Biol (2019) 1190:323-31. doi:10.1007/978-981-32-9636-7-20

57. Hu J, Jolkkonen J, Zhao C. Neurotropism of SARS-CoV-2 and its neuropathological alterations: Similarities with other coronaviruses. Neurosci Neurobehav Rev (2020) 119:184-93. doi: 10.1016/ j.neubiorev.2020.10.012

58. Song E, Zhang C, Israelow B, Lu P, Weiszman O-E. Liu F, et al. Neuroinvasion of SARS-CoV- 2 in human and mouse brain. J Exp Med (2021) 218(3): e20202135. doi: 10.1101/2020.06.25.169946

59. Sanclemente-Alaman I, Moreno-Jiménez L, Benito-Martín MS, CanalesAguirre A. Matías-Guiu JA, Matías-Guiu J, Gómez-Pinedo U. Experimental models for the study of central nervous system infection by SARS-CoV-2. Front Immunol (2020) 11:2163. doi: 10.3389/fimmu.2020.02163

60. Meinhardt J, Radke J, Dittmayer C, Franz J, Thomas C, Mothes R, et al. Olfactory transmucosal SARS-CoV-2 invasion as port of central nervous system entry in individuals with COVID-19. Nat Neurosci (2020) 24:168-75. doi: 10.1038/s415993-020-00758-5

61. Divani AA, Andalib S, Biller J, Di Napoli M, Moghimi N, Rubinos CA, et al. Central nervous system manifestations associated with COVID-19. Curr Neurol Neurosci Rep (2020) 20:60. doi: 10.1007/s11910-020-01079-7

62. Varga Z, Flammer AJ, Steiger P, Haberecker M, Andermatt R, Zinkernagel AS, et al. Endothelial infection and endotheliitis in COVID-19. Lancet (2020) 395:1417-8. doi: 10.1016/S0140-6736(20)30937-5

63. von Weyhern $\mathrm{CH}$, Kauffmann I, Neff F, Kremer M. Early evidence of pronounced brain involvement in fatal COVID-19 outcomes. Lancet (2020) 395:e109. doi:10.1016/S0140-6736(20)31282-4

64. Solomon IH, Normandin E, Bhattacharyya S, Mukerji SS, Keller K, Ali AS, et al. Neuropathological features of Covid 19. N Engl J Med (2020) 383:989-92. doi: 10.1056/NEJMc2019373 
65. Matschke J, Lütgehetmann M, Hagel C, Sperhake JP, Schröder AS , Edler C, et al. Neuropathology of patients with COVID-19 in Germany. A postmortem case series. Lancet Neurol (2020) 19:919-29 . doi: 10.1016/S1474442(20)30308-2

66. Zubair AS, McAlpine LS, Gardin T, Farhadian S, Kuruvilla DE, Spudich S. Neuropathogenesis and neurologic manifestations of the coronaviruses in the age of coronavirus disease 2019: A review. JAMA Neurol (2020) 77:1018-27. doi: 10.1001/jamaneurol.2020.2965

67. Guadarrama-Ortiz P, Choreño-Parra JA, Sánchez-Martínez CM, PachecoSánchez FJ, Rodríguez-Nava AI, Gabriela García-Quintero G. Neurological aspects of SARS-CoV-2 infection: Mechanisms and manifestations. Front Neurol (2020) 11:1039. doi: 10.3389/fneur.2020.01039

68. Conti P, Ronconi G, Caraffa A, Gallenga C, Ross R, Frydas I, et al. Induction of pro-inflammatory cytokines (IL-1 and IL-6) and lung inflammation by coronavirus-19 (COVID-19 or SARS-CoV- 2): Anti-inflammatory strategies. J Biol Regul Homeost Agents (2020) 34:2. doi: 10.23812/CONTI-E

69. Kany S, Vollrath JT, Relja B. Cytokines in inflammatory disease. Int J Mol Sci (2019) 20:23. doi: 10.3390/ijms20236008

70. Wan Y, Shang J, Graham R, Baric RS, Li F. Receptor recognition by the novel coronavirus from Wuhan: An analysis based on decade-long structural studies of SARS coronavirus. J Virol (2020) 94:7. doi: 10.1128/jvi.00127-20

71. Metha P, McAuley DF, Brown M, Sanchez E, Tattersall RS, Manson JJ, et al. COVID-19: Consider cytokine storm syndromes and immunosuppression. Lancet (2020) 395:1033-4. doi: 10.1016/S0140-6736(20)30628-0

72. Blackburn KM, Wang C. Post-infectious neurological disorders. Therap Adv Neurol Dis (2020) 13:1-17. doi: 10.1177/1756286420952901

73. Boziki MK, Mentis A-FA, Shumilina M, Makshakov G, Evdoshenko E, Grigoriadis N. COVID-19 immunopathology and the central nervous system: Implications for multiple sclerosis and other autoimmune diseases with associated demyelination. Brain Sci (2020) 10:345. doi: 10.3390/ brainsci10060345

74. Poser CM. Román GC, Emery ES $3^{\text {rd }}$. Recurrent disseminated vasculomyelinopathy. Arch Neurol (1978) 35:166-70. doi: 10.1001/ archneur.1978.00500270048010

75. Mancera-Páez O, Román GC, Pardo-Turriago R, Rodríguez Y, Anaya JM. Concurrent Guillain- Barré syndrome, transverse myelitis and encephalitis postZika: A case report and review of the pathogenic role of multiple arboviral immunity. J Neurol Sci (2018) 395:47-53. doi: 10.1016/j.jns.2018.09.028

76. Karussis D, Petrou P. The spectrum of post-vaccination inflammatory CNS demyelination. Autoimmun Rev (2014) 13:215-24. doi: 10.1016/ j.autrev.2013.10.003

77. Toro G, Vergara I, Román GC. Neuroparalytic accidents of antirabies vaccination with suckling mouse brain vaccine. Clinical and pathologic study of 21 cases. Arch Neurol (1977) 34:694-700. doi: 10.1001/archneur. 1977.00500230064011

78. Transverse Myelitis Consortium Working Group. Proposed diagnostic criteria and nosology of acute transverse myelitis. Neurology (2002) 59:499-505. doi: 10.1212/WNL.59.4.499

79. Messacar K, Schreiner TL, Van Haren K, Yang M, Glaser CA, Tyler KL, et al. Acute flaccid myelitis: A clinical review of US cases 2012-2015. Ann Neurol (2016) 80:326-38. doi: 10.1002/ana.24730

80. Kidd S, Lopez A, Nix WA, Anyalechi G, Itoh M, Yee E, et al. Clinical characteristics of patients with confirmed acute flaccid myelitis, United States, 2018. MMWR Morb Mortal Wkly Rep (2020) 69:1031-8. doi: 10.15585/ mmwr.mm6931e3:10.15585/mmwr.mm6931e3

81. Majer A, McGreevy A, Booth TF. Molecular pathogenicity of enteroviruses causing neurological disease. Front Microbiol (2020) 11:540. doi: 10.3389/ fmicb.2020.00540

82. Patel AB, Verma A. Nasal ACE2 levels and COVID-19 in children. JAMA (2020) 323:2387. doi: 10.1001/jama.2020.8946
83. Murphy OC, Pardo CA. Acute flaccid myelitis: A clinical review. Semin Neurol (2020) 40:211-8. doi: 10.1055/s-0040-1705123

84. West TW, Hess C, Cree BAC. Acute transverse myelitis: Demyelinating, inflammatory, and infectious myelopathies. Semin Neurol (2012) 32:97-113. doi: $10.1055 / \mathrm{s}-0032-1322586$

85. Schonberger LB, Bregman DJ, Sullivan-Bolyai JZ, Keenlysidee RA, Ziegler DW, Retailliau HF, et al. Guillain-Barré syndrome following vaccination in the National Influenza Immunization Program, United States, 1976-1977. Am J Epidemiol (1979) 110:105-23. doi: 10.1093/oxfordjournals.aje.a112795

86. Nachamkin I, Shadomy SV, Moran AP, Cox N, Fitzgerald C, Ung H, et al. Anti-ganglioside antibody induction by Swine (A/NJ/1976/H1N1) and other influenza vaccines: Insights into vaccine-associated Guillain-Barré syndrome. J Infect Dis (2008) 198:226-33. doi: 10.1086/589624

87. Akkad W, Salem B, Freeman JW, Huntington MK. Longitudinally extensive transverse myelitis following vaccination with nasal attenuated novel influenza A(H1N1) vaccine. Arch Neurol (2010) 67:1018-20. doi: 10.1001/ archneurol.2010.167

88. Sato N, Watanabe $\mathrm{K}$, Ohta $\mathrm{K}$, Tanaka $\mathrm{H}$. Acute transverse myelitis and acute motor axonal neuropathy developed after vaccinations against seasonal and 2009 A/H1N1 influenza. Intern Med (2011) 50:503-7. doi: 10.2169/ internalmedicine. 50.4340

89. Arcondo MF, Wachs A, Zylberman M. . Mielitis transversa relacionada con vacunación anti-influenza A (H1N1) [Transverse myelitis associated with anti-influenza A (H1N1) vaccination]. Medicina (Buenos Aires) (2010) 71:161-4.

90. Van Ussel I, Boer W, Parizel P, Cras P, Jorens PG. Encephalitis related to H1N1 vaccination: Case report and review of the literature. Clin Neurol Neurosurg (2014) 124:8-15. doi: 10.1016/j.clineuro.2014.06.003

91. Orgogozo J-M, Gilman S, Dartigues J-F, Laurent B, Puel M, Kirby LC, et al. Subacute meningoencephalitis in a subset of patients with $\mathrm{AD}$ after $\mathrm{Ab} 42$ immunization. Neurology (2003) 61:46-54. doi: 10.1212/ 01.WNL.0000073623.84147.A8

92. Ferrer I, Boada Rovira M, Sánchez Guerra ML, Rey MJ, Costa-Jussa F. Neuropathology and pathogenesis of encephalitis following amyloid beta immunization in Alzheimer's disease. Brain Pathol (2004) 14:11-20. doi: 10.1111/j.1750-3639.2004.tb00493.x

93. Kerr DA, Ayetey H. Immunopathogenesis of acute transverse myelitis. Curr Opin Neurol (2002) 15:339-47. doi: 10.1097/00019052-200206000-00019

94. Graber JJ, Allie SR, Mullen KM, Jones MV, Wang T, Krishnan C, et al. Interleukin-17 in transverse myelitis and multiple sclerosis. J Immunol (2008) 196:124-32. doi: 10.1016/j.jneuroim.2008.02.008

95. Vera-Lastra O, Medina G, Cruz-Dominguez MDP, Jara LJ, Shoenfeld Y. Autoimmune/ inflammatory syndrome induced by adjuvants (Shoenfelds' syndrome): Clinical and immunological spectrum. Expert Rev Immunol (2013) 9:361-73. doi: 10.1586/eci.13.2

96. Agmon-Levin N, Kivity S, Szyper-Kravitz M, Shoenfeld Y. Transverse myelitis and vaccines: A multi-analysis. Lupus (2009) 18:1198-204. doi: 10.1177/ 0961203309345730

Conflict of Interest: The authors declare that the research was conducted in the absence of any commercial or financial relationships that could be construed as a potential conflict of interest.

Copyright ( 2021 Román, Gracia, Torres, Palacios, Gracia and Harris. This is an open-access article distributed under the terms of the Creative Commons Attribution License (CC BY). The use, distribution or reproduction in other forums is permitted, provided the original author(s) and the copyright owner(s) are credited and that the original publication in this journal is cited, in accordance with accepted academic practice. No use, distribution or reproduction is permitted which does not comply with these terms. 